Results: Post-operatively, both patients recovered well with no complications and audiometry showed no conductive hearing loss and no worsening of bone conduction. Both patients remain under long-term follow-up.

Conclusion: EECC can present with minimal clinical findings and hearing loss but can be very extensive which could lead to serious complications if left undetected for a long time. CT scanning is extremely helpful in assessing the extent of the disease and for assisting in pre-operative planning.

doi:10.1017/S0022215116006939

\section{ID: IP197}

Role of mastoid pneumatisation in paediatric cholesteatoma

Presenting Author: Gautam Singh

Gautam Singh

Lady Hardinge Medical College \& Associated Hospitals, New Delhi

Learning Objectives: Study evaluates the factor of mastoid pneumatisation w.r.t paediatric cholesteatoma in accordance with "Evidence based medicine". Pneumatisation of mastoid in children was found to be associated with extensive and recurrent cholesteatoma in our study.

Objectives: To study the correlation between mastoid pneumatisation and cholesteatoma in paediatric patients.

Materials \& Methods: In a retrospective study design, the medical records of all the paediatric patients which underwent mastoid surgery for chronic suppurative otitis mediacholesteatoma disease were examined in the specific time period of: Jan 2010 to Jan 2016. The surgical pathology was correlated with the CT scan of the mastoid. Further, recurrence of cholesteatoma was also studied. Data was tabulated and statistically analysed.

Results: A total of 56 paediatric patients were evaluated, out of which 6 had sclerotic mastoid, 17 had diploic mastoid and 33 had pneumatic mastoid. Statistical evaluation of this data was found to be significant thereby implying that cholesteatoma development is more in pneumatic mastoid. Further sinus tympani and facial recess involvement was also significantly more in a pneumatised mastoid. Evaluation of our records further revealed that recurrence of paediatric cholesteatoma is also more in pneumatic mastoid.

Conclusions: It is thus hypothesised that a well pneumatised mastoid may lead to cholesteatoma. In addition, cholesteatoma is more extensive and might recur in a pneumatised mastoid in children.
doi:10.1017/S0022215116006940

\section{ID: IP198}

Role of "Paediatric Tympanoplasty" in modern otology

Presenting Author: Gautam Singh

Gautam Singh

Lady Hardinge Medical College \& Associated

Hospitals, New Delhi

Learning Objectives: Paediatric tympanoplasty is a controversial subject with no consensus amoung otologists regarding the minimum age for the surgery. Moreover the subject does not find mention in standard text. There is a general tendency across the world to delay the said surgery on account of various factors like: eustachian tube maturity, size and site of perforation \& age etc. I would be examining the concept of "Paediatric Tympanoplasty" in accordance with evidence based medicine: two prospective studies carried out at LHMC \& Associated Hospitals, New Delhi. Our results indicate that the graft uptake in children is equal to adults and addresses the issue of deafness in children.

Objective: To evaluate the success rate of tympanoplasty type $I$ in a select pediatric age group of 5 to 8 years, and thereby analyse the factors suggested to influence the outcome of the said surgery.

Study Design: A prospective study with control. 60 patients of chronic suppurative otitis media-inactive mucosal disease of either sex were divided into two groups of 30 each; Group A [paediatrics, 5-8years] and Group B [adults, $>14$ years]. All the patients underwent tympanoplasty type I by underlay technique using temporalis fascia graft. Pre-operative tympanometric volume was recorded in all the cases and analysed with the graft uptake results post-operatively. The data pertaining to surgical and audiological success was tabulated and statistically analysed.

Results: We recorded an impressive surgical success rate of $87 \%$ in Group A compared to a $90 \%$ success rate for group B. Also, an audiological success of $69 \%$ and $78 \%$ was recorded in the pediatric and adult group, respectively. Statistical analysis of the data revealed that age, status of the contralateral ear (taken as a measure of function of the Eustachian tube) and size of the perforation are not significant factors which might influence the outcome of paediatric tympanoplasty, but the site of the perforation was found to be a significant factor for the said surgery with anterior perforations recording a poor success rate. On the basis of mean tympanometric volume of $1.6 \mathrm{~cm} 3$ the patients were divided into two groups: in Group A (tympanometric volume $<1.6 \mathrm{~cm} 3$ ), and group B (tympanometric volume $>1.6 \mathrm{~cm} 3$ ). A graft uptake of $95 \%$ and $77 \%$ was recorded in Group A \& B 
respectively. However, the statistical evaluation of the data revealed no significant effect of this factor.

Conclusions: Tympanoplasty type I with underlay grafting using temporalis fascia in children aged between 5 to 8 years, gives good anatomical and functional results.

doi:10.1017/S0022215116006952

\section{ID: IP199}

Tympanic impedance measurement with standardised nasopharyngeal air pressures - a new test of Eustachian tube function

\section{Presenting Author: Matthew E. Smith}

Matthew E. Smith, James R. Tysome

Cambridge University Hospitals NHS

Foundation Trust

Learning Objectives: To understand a novel method of measuring ET dysfunction.

Introduction: Eustachian tube (ET) dysfunction is a common and varied condition with significant associated morbidity. In most cases it is caused by a failure of the ET to adequately open, however there is currently no reliable method of assessing this opening. Tubomanometry is a test that has recently shown good inter-individual repeatability as a measure of ET opening and function, by measuring middle ear pressure after the application of regulated nasopharyngeal pressures during swallowing. We present the first reports of a novel test: middle ear impedance measurements during standardised nasopharyngeal positive pressure bursts (tuboimpedance). We assess repeatability in healthy ears, and whether this new assessment provides any advantages over tubomanometry.

Methods: Ethical approval was obtained. Tubomanometry and tuboimpedance assessments were performed in 20 screened, healthy ears (10 volunteers). Each ear underwent tests while the patient swallowed a water bolus during applied nasopharyngeal pressures of $20,30,40$ and $50 \mathrm{mbar}$. Immediate and delayed repeats were performed at each pressure.

Results: ET opening was detected more frequently with the tuboimpedance method, with a $100 \%$ detection rate using a nasopharyngeal pressure of $30 \mathrm{mbar}$ or more. ET opening at $20 \mathrm{mbar}$ was detected more frequently with tuboimpedance. Repeatability of both tests, as measured by Intraclass Correlation Coefficient, was very good for both immediate and delayed repeats. Repeatability for the tubomanometry $\mathrm{R}$ value was mixed.

Conclusions: Tuboimpedance may provide a repeatable measure of ET opening that is easier to perform, due to lower required nasopharyngeal pressures and fewer issues with poor ear-probe sealing. Further assessment in patients with different forms of ET dysfunction is required.
doi:10.1017/S0022215116006964

\section{ID: IP200}

Petrous bone cholesteatoma: our recent experience

Presenting Author: Tommaso Sorrentino

Tommaso Sorrentino, Nader Nassif, Francesco Mancini, Luca Oscar Redaelli DeZinis

Spedali Civili Brescia

Learning Objectives:

Introduction: Petrous bone cholesteatoma is a rare condition affecting the temporal bone. It can be congenital or an evolution of a middle ear cholesteatoma. Usually at clinical presentation it involves labyrinth, facial nerve or vascular strucures. We presented 7 cases of petrous bone cholesteatoma treated in our department in the last 4 years.

Methods: We retrospectively review 7 cases of temporal bone cholesteatoma recently treated in our department.

Results: Three patients presented a previous hystory of middle ear surgery negleted and they presented at the consultation for pain in ear region. Two had already total hearing loss on that side. In the other 4 cases the cholesteatoma was congenital and the diagnosis was made by magnetic resonance for facial palsy in three cases and paralysis of the VI nerve in one case. The surgical approach was transcochlear in 2 cases, translabyrinthine in 4 cases and 1 patients was treated by supralabyrinthine approach. Facial nerve was interrupted in one case and an end to end anasthomosis was performed. We were able to preserve hearing in only 1 case. Facial nerve function improve but normalized only in 1 case.

Conclusions: Facial nerve fuction is the challenging problem in case of petrous bone cholesteatoma. Hearing can be preserved only in case of supralabyrinthine extension.

doi:10.1017/S0022215116006976

\section{ID: IP201}

Summarising cholestaetoma surgery and A new method of closing the mastoid cavity

Presenting Author: Pankaj Srivastava

Pankaj Srivastava $^{1}$, Rohit Mehrotra ${ }^{2}$

${ }^{1}$ Pankaj ENT hospital, ${ }^{2}$ mehrotra ENT hospital

Learning Objectives:

Anatomically there are two separate layers in ear, first middle ear cleft comprising mastoid air cells, antrum, aditus, middle ear and Eustachian tube all lined by contiguous mucosa which secretes mucus and is drained finally to 